\title{
A computer system for processing eye-movement records*
}

\author{
HAROLD L. KUNDEL and CALVIN F. NODINE \\ Temple University, Philadelphia, Pennsilvania 19140
}

A computer system using the LINC-8 computer was developed to process eye-movement records from two $16-\mathrm{mm}$ cine film input sources: the Mackworth stand camera and the Mackworth wide-angle camera. Data reduction was accomplished by projecting the films onto an $\mathrm{X}-\mathrm{Y}$ plotting table. An operator marked, using a cursor, either the eyespot, in the case of the stand camera, or the pupil and display center, in the case of the wide-angle camera, in order to digitize the locus of the gaze. This information was normalized and stored on magnetic tape as: ordinal fixation number, fixation duration, and fixation coordinates ( $\mathrm{X}$ and $\mathrm{Y}$ ). $\mathrm{A}$ software library was created to analyze the three eye-movement measures. One program calculates interfixation distance, fixation time, and dispersion of fixations about a point. A second program operates on data matrices to: calculate area covered by a fixation pattern, frequency of redundant fixations in a fixation pattern, and distribution of fixations over features of the visual display. Data are displayed on either scope or the Calcomp plotter.

For the researcher who studies eye movements using cinematographic techniques, data reduction can be the most laborious phase of research. Each frame of cine film must be inspected in order to determine the location of the eye relative to the visual display. If the eye has been filmed at usual rates of $8-10$ rames/sec, the number of frames the researcher must inspect accumulates rapidly, limited only by the amount of viewing time given the $\mathrm{S}$. Because of the vast amount of data generated in eye-movement studies and the requirement that the eye position be located with a high degree of accuracy, the computer was the natural choice of a partner to assist the research with the task of data reduction.

The present paper documents the authors' experiences in using a computer system to help with the task of data reduction and analysis of eye-movement records from 16-mm cine film. Eye-movement records were obtained from two types of eye cameras, the Mackworth stand camera, which measures the gaze of the $S$ by superimposing a light spot reflected from the cornea onto the stimulus display, and the Mackworth wide-angle camera, which measures the gaze of the $S$ by superimposing a reflected image of the stimulus display onto the pupil of the eye.

A detailed account of the recording principles for the stand camera can be found in Mackworth (1967), and for the wide-angle camera in Mackworth (1968).

\section{DATA ACQUISITION}

Figure 1A shows a frame of cine film generated from the stand camera, and Fig. 1B shows a frame generated from the wide-angle camera. The visual display in these pictures was a $2-\mathrm{cm}$ white square with a black surrounding border, $1 \mathrm{~cm}$ wide. A dot was located in the center of the white square, and dots were positioned $1 \mathrm{~cm}$ from outside edges of the black border on both horizontal and vertical axes with respect to the center dot. Each dot, including the center one, served as targets in a calibration study. The targets were viewed at a distance of $35 \mathrm{~cm}$. Thus, each target dot was displaced $4 \mathrm{~cm}$ or $6 \mathrm{deg}$ in visual angle from center. The procedure with the stand camera was to present the display on a display board to the $S$, who was restrained by a biteboard and foreheadrest. A typical calibration record in which the $S$ fixates on the center dot and each of the four border dots is shown in Fig. 2.

The procedure for the wide-angle camera was to rear-project the display. Again, the S was restrained, but by using a combination chin- and foreheadrest rather than biteboard. It should be noted that the center of the
Fig. 1. One frame of $16-\mathrm{mm}$ cine film recorded by stand camera, showing reflected eye spot on target display (A), and one frame of film recorded by a wide-angle camera, showing reflected image of target display on pupil (B).
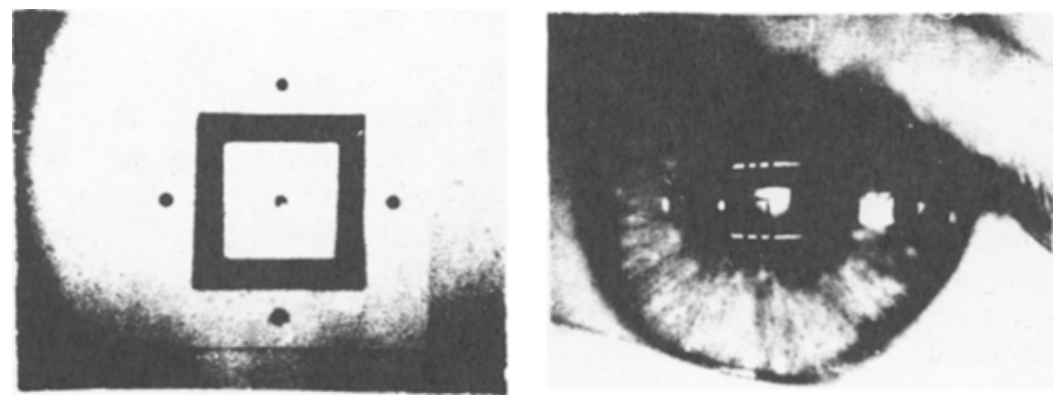

* This research was supported, in part, by Research Grant GM 14548-07, NIGMS. Reprint requests should be addressed to the Diagnostic Radiology Research Laboratory, Temple University School of Medicine. Philadelphia, Pennsylvania 19140 . 


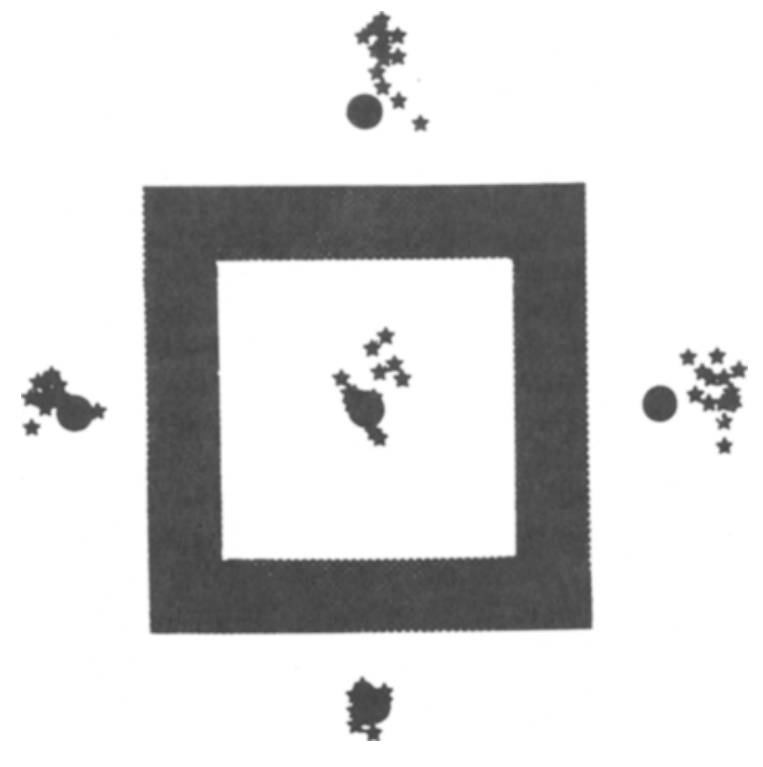

Fig. 2. Calibration record of S F S. to target display recorded using stand camera.

pupil is used as the reference point for determining the fixation point on the display with this recording system.

In addition to the two methods of presenting stimulus materials described above, television displays have been employed as a third method of presentation. The television images, in this case are stored on a 200-channel videodisk recorder. Presentation of the television display is controlled by a programmable digit logic module (BRS/LVE), which also automatically turns the camera motor on and off as well as marking the film with a signal light.

\section{DATA REDUCTION AND STORAGE General Procedure}

The 16-mm cine films from either camera are projected from a Vangard projector, frame by frame, onto the surface of an X-Y plotting table (Thomson Pencil Follower) that interfaces with a LINC.8 computer. Figure 3 shows a schematic diagram of the data-processing system. It has been described in detail in Stewart et al (1969).

After appropriate initializing procedures (locating the center of the display with reference to the Thomson table and adjusting magnification and correction factors), an operator enters the data from each frame of cine film, using a cursor. The coordinates of interest are put into $X-Y$ digital notation and entered in the computer, after which the cine film is automatically advanced to the next frame.

\section{Data Reduction}

In the case of the stand camera, the operator, after locating the center of the display. follows the reflected "eyespot" with the cursor. Entry of the data from the wide-angle camera is somewhat more complicated, since both the position of the pupil and the position of the reflected image on the pupil change with each frame because the shape of the cornea is not truly spherical and the motion of the eye is not linear. Here, the operator first enters the center of the display by aligning the cursor with an outline of the display drawn on the cursor, and then enters the center of the pupil by aligning it with a series of concentric rings outlined on the cursor. The computer calculates the locus of the fixation as the X-Y displacement of the center of the pupil from the center of the display.

From this point onward, the data from both cameras is treated by the computer identically. The $X-Y$ coordinates of the locus of fixation on each frame are compared to the previous frame in order to determine if the axis of the gaze has shifted more than a given increment (which has been predetermined). If the shift is greater than the increment, it is scored as a new fixation; if the shift is less than the increment, it is scored as the same fixation (i.e., the locus of fixation is unchanged). The size of the increment chosen by the operator is determined by several factors: size of the reflected light spot, drift of the eye during fixation, fine saccades, and out-of-focus films which make it difficult to locate the pupil boundary and stimulus display center. The value of the increment can be determined by measuring the mean variation in computed fixation locus while the $\mathrm{S}$ steadily fixates a given point on the stimulus display.

\section{Data Storage}

The data are stored on magnetic tape in the form: ordinal fixation number, fixation duration, $\mathrm{X}$, and $\mathrm{Y}$. The fixation duration is stored as number of frames. Accuracy of fixation times is dependent on accuracy of the camera frame rate. The $\mathrm{X}$ and $\mathrm{Y}$ coordinates are stored half size in centimeters with respect to the stimulus display.

\section{Sources of Error and Correction Factors}

The calibration pattern in Fig. 2 was produced by asking the $S$ to fixate on the center dot of the test

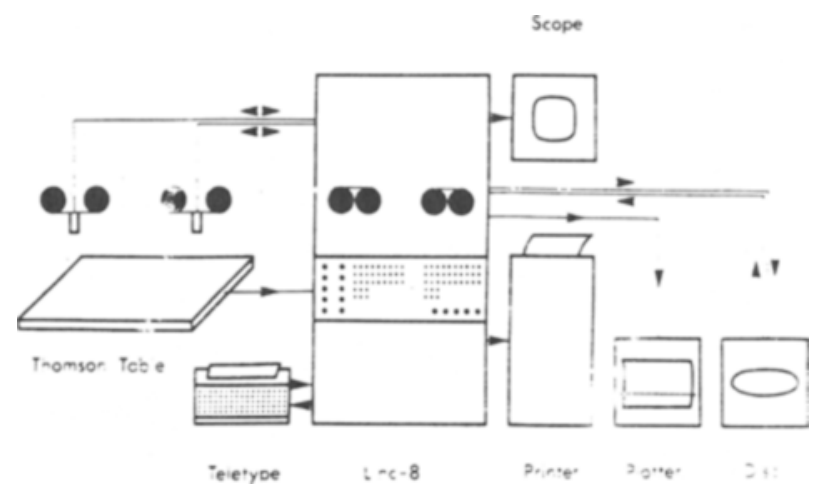

Fig. 3. Schematic diagram of data processing system showing Vangard precision projectors, Thomson table, and LINC-8 computer plus standard peripherals. 
Table 1

Mean Precision and Registration Accuracy for the Stand Camera and the Wide-Angle Camera

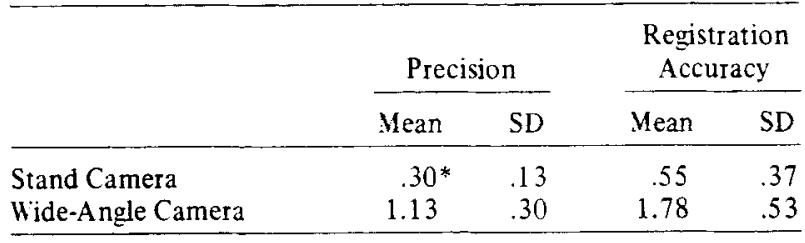

*Data reported in degrees of visual angle, based on three $S$ s for each condition, fixating a central point and four peripheral points three times each. See text for description of target dispiay.

pattern. The stand camera was then adjusted to bring the reflected "eyespot" as close to this point as possible. After this, the $\mathrm{S}$ was asked to fixate the center, left, top, right, and bottom dots over and over while the E filmed the eye.

If preliminary inspection of the filmed calibration pattern that precedes the experiment proper indicates close alignment of the eyespot with the calibration points, then the data is scored directly. If, however, the eyespot does not line up with the calibration points (as in Fig. 2, where the eyespot falls outside of the left and right dots on the horizontal axis due to avoidance of the reflected eyespot or astigmatism on the part of the $S$ ), a correction can be applied to bring the eyespot in line with the calibration points. This correction is calculated by multiplying all points to the right or left of center by an adjustment factor [(true distance from center)/(measured distance from center)]. The simple linear correction is not ideal, since error is nonlinear, but as a first-order approximation it brings the eyespot into reasonable alignment. A similar correction procedure can be applied to align pupil center with display center when the wide-angle camera is used. Four correction factors are possible with the programs described below: $\mathrm{X}-, \mathrm{Y}+$ $\mathrm{X}+, \mathrm{Y}-$. If corrections are used, they should be checked after each run and required adjustments made. Gould and Shaffer (1965) have reported a somewhat more complex scheme for correcting this sort of error.

Misalignment of the eyespot with calibration points on the display is one source of error which can be easily detected and corrected, as noted above. Another source of error, which is more difficult to detect, is head movement. Comparison of calibration points taken at the beginning and end of a run will indicate whether head movement occurred during filming. If the beginning and end calibration points differ by more than some acceptable amount, then the run should be rejected. With the stand camera, differences between beginning and end calibration points greater than $1 \mathrm{deg}$ of visual angle will result in the rejection of $50 \%$ of the runs. Head movement is less serious a problem with the wide-angle camera (Mackworth, 1968).

A final source of error is introduced by the operator

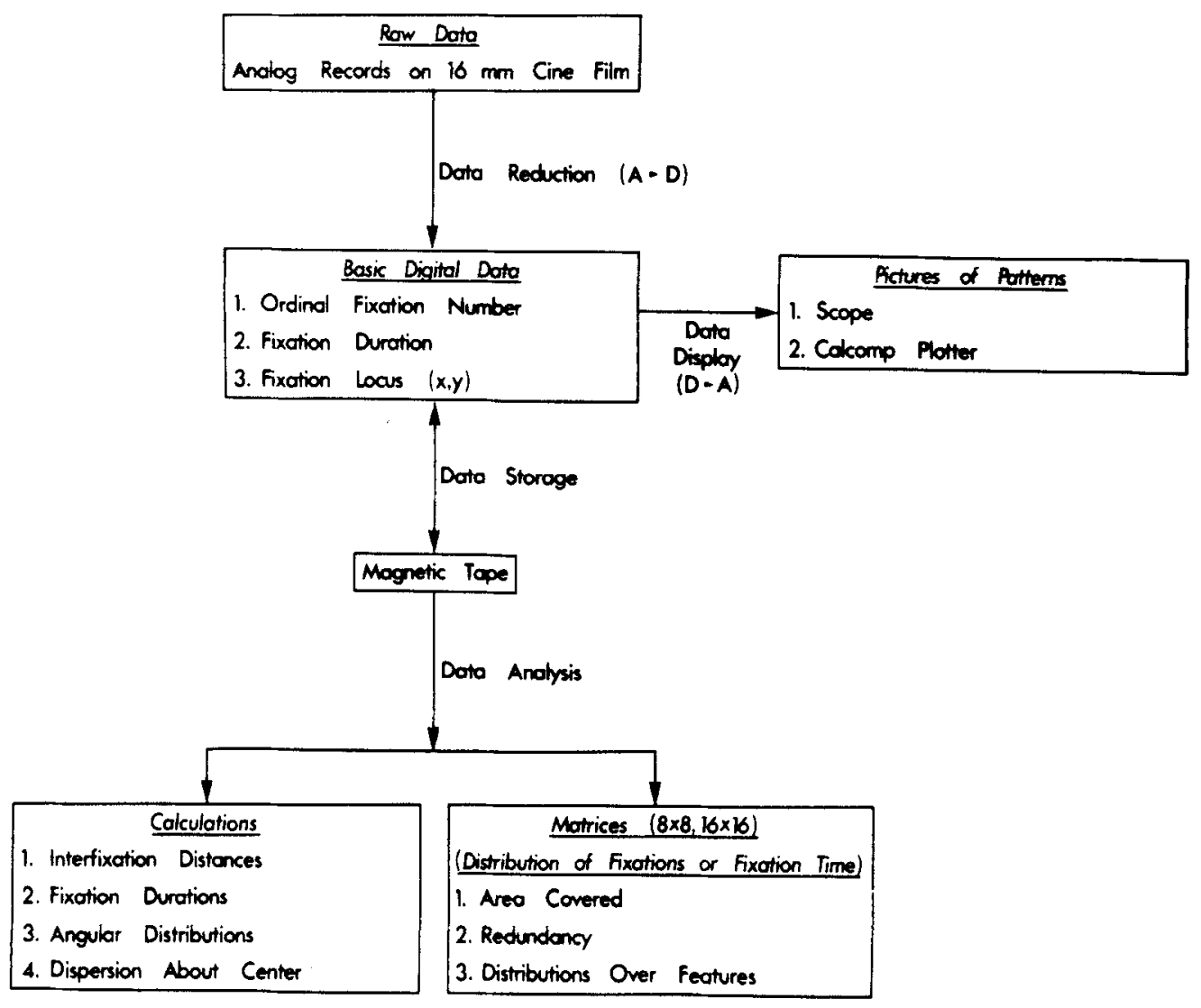

Fig. 4. Flow chart summarizing data-processing system for eye-movement records. 

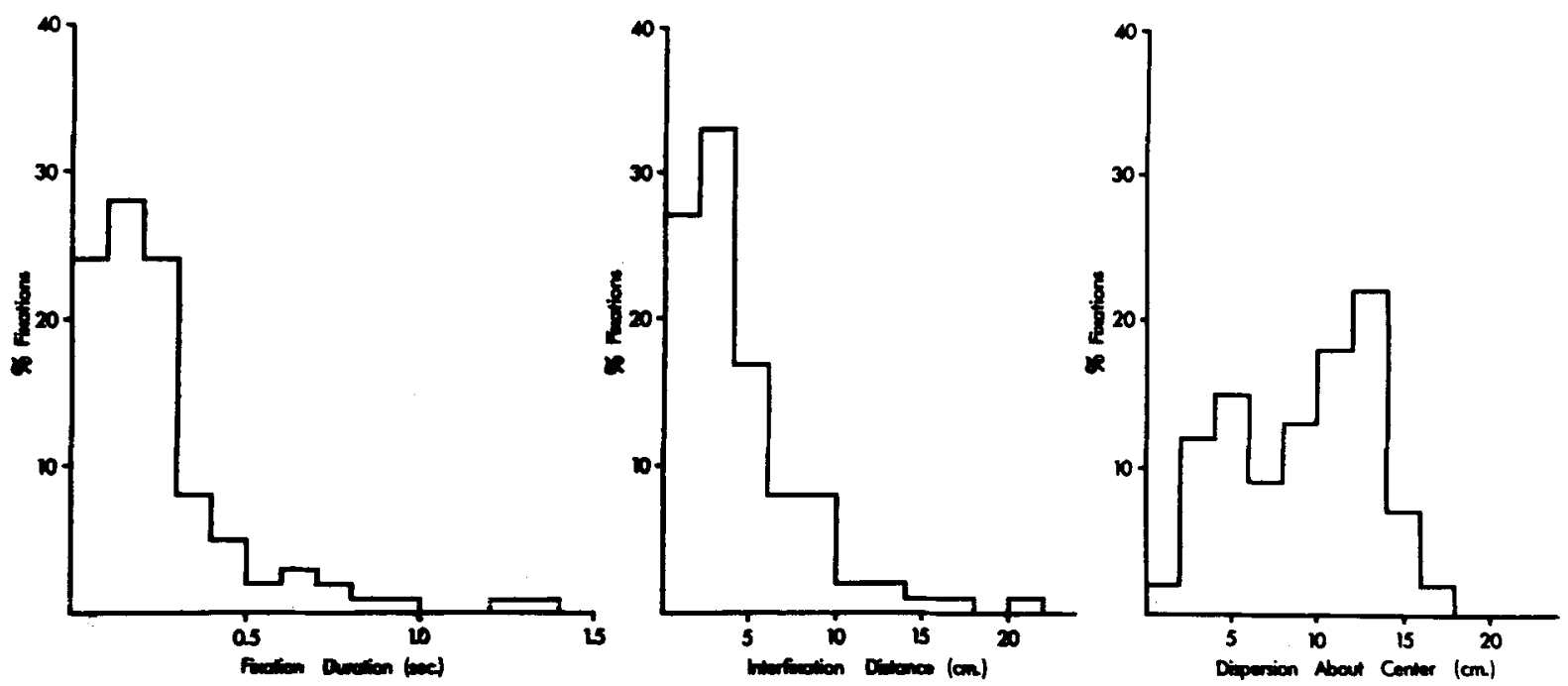

Fig 5. Histograms showing percent fixations as a function of fixation duration (left), interfixation distance (center), and dispersion sbout display center (right) for S F.S. viewing chest X-ray (Fig. 8A).

entering the data. Judgments concerning the location of the center of the pupil, center of the display, and center of the eyespot are all subject to error. Table 1 provides a comparison of precision and registration accuracy of the stand camera and the wide-angle camera based on calibration data on three Ss.

\section{DATA ANALYSIS}

Once the data are stored in digital form on magnetic tape, a large variety of analyses can be performed. The analyses that the authors are currently using fall into two categories: those concerned with summarizing the data using descriptive statistics, and those concerned with transforming fixations into matrix format. They are summarized in Fig. 4.

\section{Descriptive Statistics}

One program calculates interfixation distance, frequency of redundant fixations, the dispersion of fixations about any preselected point, and amount of display area covered by the fovea. These parameters are calculated as running means and standard deviations. Histograms of fixation time, interfixation distance, and fixation dispersion about a preselected point are also calculated. Figure 5 presents histograms showing percent fixations as a function of each of these paramters. These data were obtained from one $S$ tested using the stand camera; however, it is possible to derive these parameters by summing over many $\mathrm{Ss}$.

\section{Matrix Operations}

A second program utilizes data matrices to calculate total fixations and total fixation time spent in any matrix element or combination of elements. Figure 6 presents data in two 16 by 16 matrices, showing distribution of fixations. Both resolution and sequence information are lost when the data are transferred to matrix form. Each cell of the 16 by 16 matrix represents $4 \mathrm{~cm}^{2}$ of display space $(2 \times 2 \mathrm{~cm})$.

As the data are sequentially entered into the matrix, a measure of fixation redundancy is calculated. A fixation is considered redundant if it falls into a matrix cell already occupied by a previous fixation. The proportion of redundant fixations in the data shown in Fig. 6A was .52 , for example. The cumulative proportion of the total area sampled by the fovea is also calculated by mexsuring the propertion of alls accupied as the data

Table 2

Distribution of Fixations to Each Matrix Partition for a Chest X-Ray

\begin{tabular}{lc}
\hline Matrix Partitions & $\begin{array}{c}\text { Proportion } \\
\text { of Fixations }\end{array}$ \\
\hline Left Lung & .16 \\
Right Lung & .29 \\
Heart and Diaphragm & .14 \\
Prominent Edges & .37 \\
Periphery & .03 \\
\hline
\end{tabular}

Table 3

Distribution of Fixations to Each Matrix Partition for the Letter Pair EF

\begin{tabular}{lcc}
\multicolumn{1}{c}{ Matrix Partitions } & $\begin{array}{c}\text { Proportion of } \\
\text { Fixations }\end{array}$ & $\begin{array}{c}\text { Fivation } \\
\text { Time }\end{array}$ \\
\hline Common Letter Outline & .23 & .30 \\
$\begin{array}{l}\text { Distinctive Features } \\
\quad \text { Left Letter }\end{array}$ & & \\
$\quad$ Right Letter & & \\
Spaces Within Letters & .24 & .28 \\
$\quad$ Left Letter & .12 & .08 \\
$\quad$ Right Letter & .18 & .17 \\
Space Around Letters & .12 & .08 \\
$\quad$ Left Surround & .12 & .08 \\
$\quad$ Right Surround & & \\
$\quad$ Off Target & &
\end{tabular}




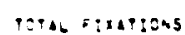
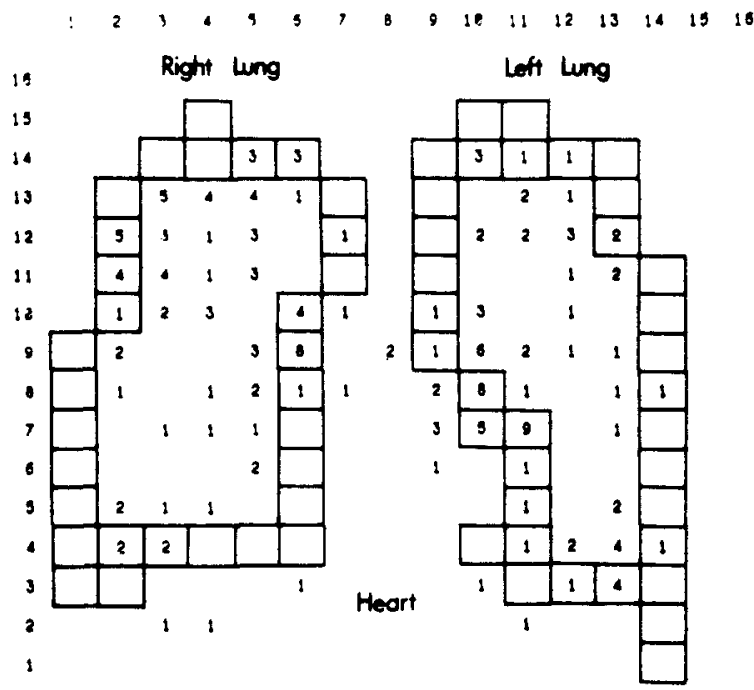

VTAL Fixaplons,

Tath Fanest
TITAE TMATITSS

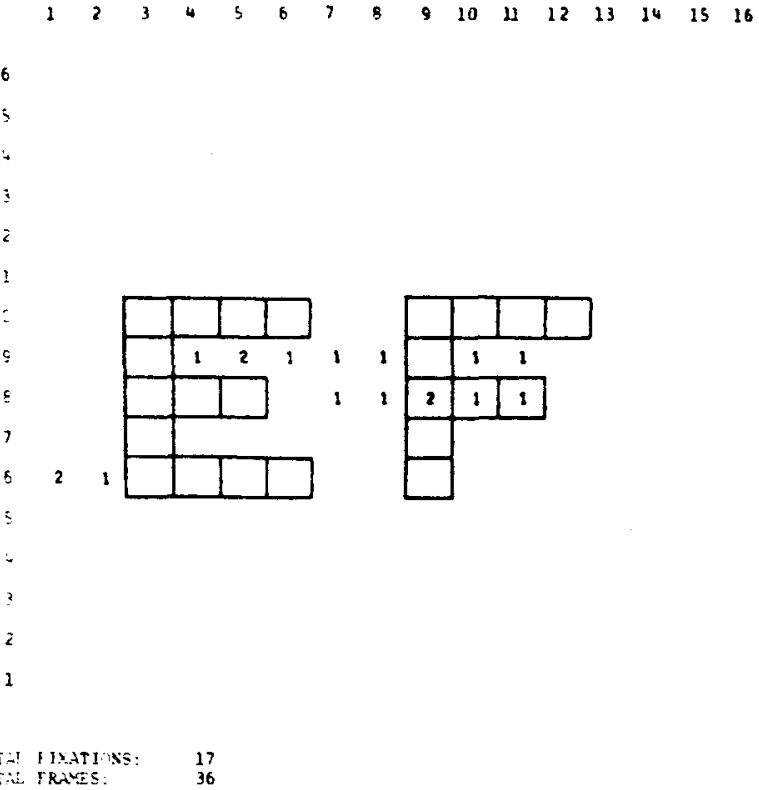

Fig. 6. A 16 by 16 data matrix showing distribution of fixations to chest X-ray (right) and letter pair EF (left).

matrix is developed fixation by fixation. A plot of this is shown in Fig. 7.

Once the data matrix is developed, a number of useful values can be calculated. For example, the matrix can be partitioned into subareas to designate features of the stimulus display, and the proportion of fixations or proportion of fixation time spent in a given subarea(s) representing a stimulus feature can be obtained. Table 2 presents data from a matrix partitioned to represent the features of a radiological image (chest X-ray), and
Table 3 presents data from a matrix partitioned to represent the features of letters EF.

\section{DATA DISPLAYS}

In addition to the summarizing of data which is handled by the data analysis programs, dynamic presentations of the data can be achieved by plotting a fixation record on either an oscilloscope or a Calcomp plotter. The main advantage in plotting the fixation record is that the sequence of fixations is made available,

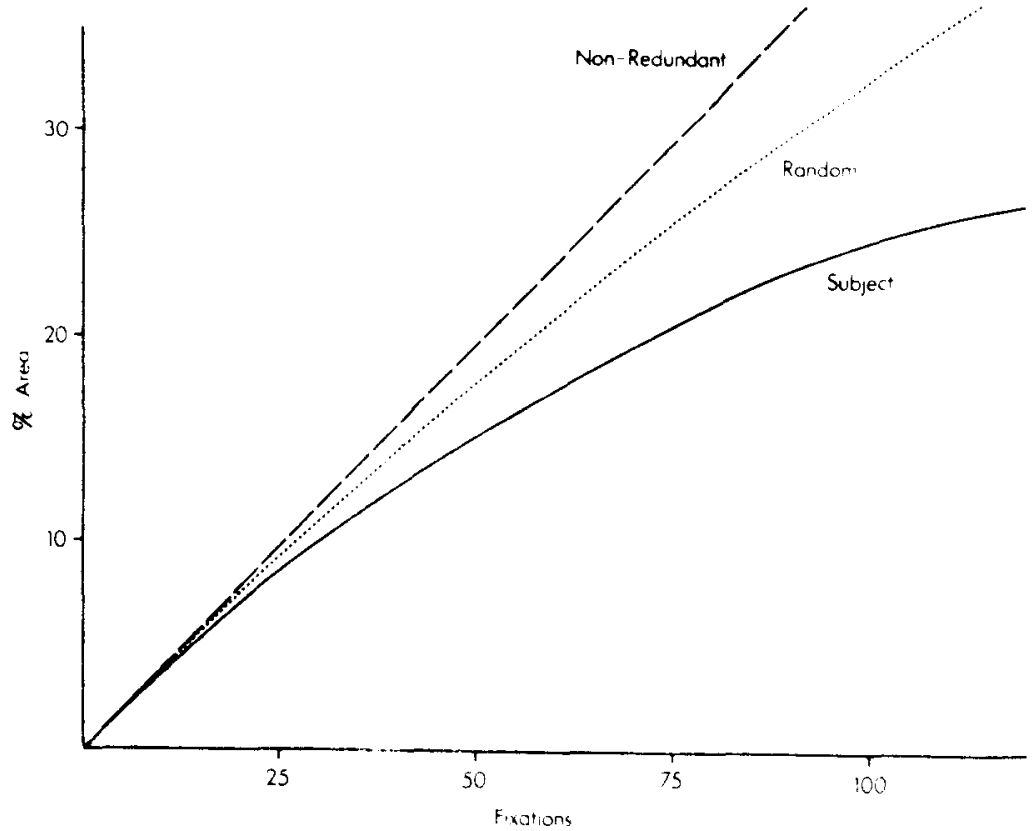

Fig. 7. Plot of S F.S. viewing chest X-ray (Fig. 8A), showing percent 16 by 16 matrix covered as a function of the cumulative number of fixations. The S's record is contrasted with the situation in which each fixation falls into a different cell of the 16 by 16 matrix (nonredundant). and the situation in which fixations fall at random into the cells of the 16 by 16 matrix (random). 
Fig. 8. Fixation record of S F.S. viewing chest X-ray (A), and S T.M. viewing letter pair EF (B).
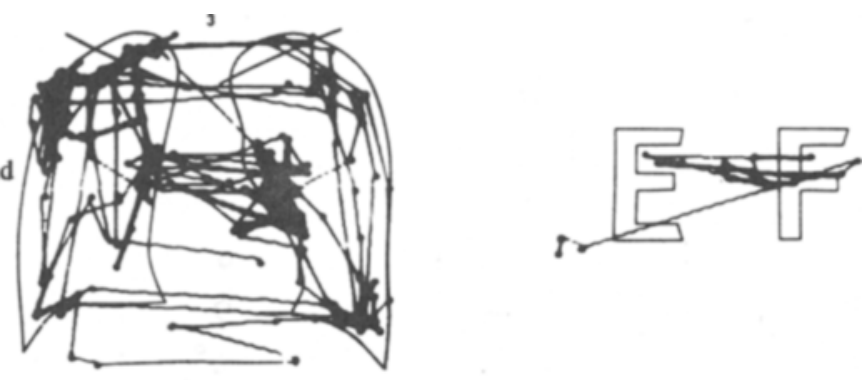

thus permitting inferences to be dirawn about the S's scanning strategy for extracting and processing information from the stimulus display (Kundel \& La Follete, 1972; Nodine \& Steuerle, in press).

The authors' program for displaying a fixation record plots successive fixations on an outline or a picture of the stimulus display and connects the fixations by a line. The fixation record is displayed on either an oscilloscope or a Calcomp plotter. The oscilloscope display is a faster and more convenient way of generating fixation records than the Calcomp plotter, since a Polaroid picture can be taken of the sequence of fixations and this can be superimposed (optically) over an outline or a picture of the stimulus display. A Tektronix Type $\mathrm{C}-12$ camera with graticule attachment is used to produce such displays. In addition to generating complete fixation records, it is possible to generate partial records (e.g., first eight fixations or Fixations 4-8) by specifying starting and ending points when calling the program.

Examples of use of the plotting program are shown in Fig. 8. Figure $8 \mathrm{~A}$ presents a fixation record of a $\mathrm{S}$ viewing a chest $\mathrm{X}$-ray, and Fig. $8 \mathrm{~B}$ presents a fixation record of a $S$ viewing a pair of letters of the alphabet.

\section{CONCLUSION}

A general system for reducing and analyzing eye-movement data recorded on $16-\mathrm{mm}$ cine film has: been discussed. The potential of this system is large, ranging from directly coupling the eye-movement recorder to the computer for on-line analysis to eliminating the $S$ altogether and developing simulation models that can be tested with the analysis programs. However, the computer is a mean to an end, not an end itself; it is a tool of the experimenter, not the experiment. The available software has been designed to answer specific experimental questions and to test specific hypotheses about the acquisition of data by the visual system. Hopefully, new analytical techniques will be devised as our understanding of eye movements increases.

\section{REFERENCES}

Gould, J. D., \& Schaffer, A. Eye-movement patterns in scanning numeric display. Perceptual \& Motor Skills, 1965, 20 , $521-535$

Kundel, H. L.. \& La Follette, P. S. Visual search patterns and experience with radiological images. Radiology, 1972, 103, 523-528.

Mackworth, N. H. A stand camera for line-of-sight recording. Perception \& Psychophysics, 1967, 2, 119-127.

Mackworth, N. H. The wide-angle reflection eye camera for visual choice and pupil size. Perception \& Psychophysics, $1968,3,32-34$.

Nodine, C. F., \& Steuerle, N. L. Development of perceptual-cognitive strategies for differentiating graphemes. Journal of Experimental Psychology, in press.

Stewart, G. H., Lynch, P. R., \& Gimenez, J. L. A versatile technique for the analysis of dynamic multidimensional information. Medical \& Biological Engineering, 1969, 7 , 435-438. 